\title{
Comparação dos comportamentos relacionados à saúde em praticantes e não praticantes da ginástica laboral
}

\author{
Comparison of health-related behaviors \\ in practitioners and non-practitioners of \\ gymnastics
}

Fisisenectus . Unochapecó Ano 4, n. 1 - Jan/Jun. 2016 p. $51-59$

Josiane Schadeck de Almeida Altemar. josianesa@unochapeco.edu.br Doutora do curso de Fisioterapia da Universidade Comunitária da Região de ChapecóUnochapecó.

Ianca Stürmer. iancasturmer@unochapeco.edu.br

Estudante do curso de Fisioterapia. Universidade Comunitária da Região de Chapecó Unochapecó.

\section{Resumo}

Introdução: Dependendo da escolha e comportamento do indivíduo perante sua saúde, o trabalho pode afetar sua qualidade de vida, que está associada ao estilo de vida do trabalhador, englobando aspectos como hábitos alimentares, alcoólicos e esportivos. Um dos programas no trabalho que visa estimular comportamentos e atitudes conscientes para uma vida saudável no âmbito ocupacional é a Ginástica Laboral (GL). Objetivo: Comparar o comportamento relacionado à saúde de funcionários praticantes e não praticantes do projeto de extensão em Ginástica Laboral de uma universidade do oeste de Santa Catarina. Materiais e métodos: Pesquisa quantitativa transversal, com 60 sujeitos de 11 setores da Universidade, sendo 30 praticantes e 30 não. Para a coleta de dados, foi utilizado um questionário autoaplicável YRBS e adaptado ao Brasil por Guedes e Lopes. 0 questionário aborda questões referentes a hábitos alimentares, higiene, sono, álcool, hábitos tabácicos, atividade física, sendo aplicado no próprio local de trabalho no mês dezembro de 2015. Resultados: Não houve diferença significativa entre os dois grupos na maioria dos itens comparados. Notou-se, entretanto, diferença nos hábitos esportivos e alcoólicos, onde se constatou que os não praticantes de GL realizam mais exercícios físicos e bebem menos se comparado aos praticantes. Conclusão: A prática de GL não influenciou nos comportamentos em saúde de praticantes desta pesquisa, fazendo-se necessários novos estudos para sua efetiva comprovação; outra frente que se abre é a necessidade do trabalho da ação extensionista direcionada para o âmbito da educação em saúde dentro do projeto, no sentido do cuidado integral.

\section{Palavras-chave}

Promoção da saúde; Estilo de vida; Saúde do trabalhador.

\section{Fisiß̌enectus}




\begin{abstract}
Introduction: Depending on the choice and individual behavior towards their health, work can affect their quality of life, which is associated with the worker's lifestyle, covering aspects such as diet, alcohol and sports. One of the programs at work aimed at stimulating behaviors and conscious attitudes for a healthy life in the occupational context is the Gymnastics (GL). Objective: To compare the behavior related to health practitioners and employees do not practice the extension project in Gymnastics at a university in western Santa Catarina. Materials and Methods: Cross-sectional quantitative research with 60 subjects of 11 sectors of the University, 30 practitioners and 30 not. To collect data, we used a self-administered questionnaire YRBS and adapted to Brazil by GUEDES and LOPES (2010). The questionnaire addresses issues regarding eating habits, hygiene, sleep, alcohol, tobacco habits, physical activity, being applied in the workplace in the month December 2015. Results: There was no significant difference between the two groups in most of the compared items. Is noted however, difference in sports and drinking habits, where it was found that non GL practitioners perform more exercise and drink less compared to practitioners. Conclusion: GL practice did not influence the health behaviors of practitioners of this research, by making further studies necessary for effective verification; another front that opens is the need of the work of extension action directed to the field of health education within the project, in the sense of comprehensive care.
\end{abstract}

\title{
Keywords
}

Health Promotion; Lifestyle; Worker's health.

\section{Introdução}

$\infty \infty \times \infty \times \infty \times \infty \times \infty \times \infty \times \infty \times \infty \times \infty \times \infty$

0 desenvolvimento tecnológico que a sociedade moderna tem vivenciado tem facilitado enormemente a vida do trabalhador. Mas, na correria do dia a dia, a população se doa cada vez mais ao trabalho, buscando aumentar sua produtividade e esquecendo-se do cuidado com a própria saúde e bem-estar biopsicossocial, acarretando no desenvolvimento de doenças ocupacionais, em decorrência dos esforços repetitivos, estresse e maior exigência no ambiente de trabalho ${ }^{1}$.

Para Katsurayama, Parente e Pires" "o trabalho é o que move a vida humana e sempre afetará a saúde, seja para conduzir a um lado bom ou para um ruim, dependendo dos processos psicodinâmicos desenvolvidos no trabalho e da escolha dos indivíduos".

Desse modo, a qualidade de vida está muito associada à escolha do estilo de vida dos indivíduos, englobando aspectos importantes, como atividade física, nutrição e relacionamento social, os quais têm sido tradicionalmente associados com questões da saúde e bem-estar ${ }^{3}$.

A saúde também deve ser vista como um recurso para a vida, não como objetivo de viver, sendo que os determinantes e condicionantes da saúde como a paz, habitação, educação, alimentação, renda, ecossistema estável, recursos sustentáveis, justiça social e equidade estejam presente para todos, para assim alcançar qualidade de vida e saúde ${ }^{4}$.

Um dos programas que visam promover comportamentos e atitudes conscientes para uma vida saudável no trabalho é a Ginástica Laboral (GL), propiciando mudança individual, que acarretará benefícios pessoais e coletivos, a partir de melhorias no ambiente ocupacional ${ }^{5,6}$.

Desse modo, indaga-se sobre os comportamentos de saúde em praticantes de $G L$, se esta leva a uma diferença positiva nos hábitos de vida e comportamento de saúde dos trabalhadores. Sendo assim, esse estudo tem como objetivo comparar os comportamentos em saúde em praticantes e não praticantes de ginástica laboral em uma Universidade do oeste catarinense.

\section{Materiais e métodos}

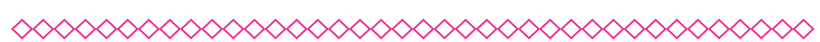

A pesquisa tem caráter quantitativo do tipo descritivo transversal. A população da pesquisa são indivíduos com vínculo empregatício em uma 
universidade do oeste de Santa Catarina, que aceitaram fazer parte da pesquisa, assinando um termo de consentimento livre e esclarecido, independentemente do gênero e idade. A amostra teve participação de 11 setores da Universidade, totalizando 60 indivíduos. Dentre estes, metade era praticante da Ginástica Laboral (GL) e a outra não.

Para a coleta de dados, foi utilizado um questionário autoaplicável adaptado do questionário YRBS e adaptado ao Brasil por Guedes e Lopes ${ }^{7}$. 0 questionário aborda questões referentes a hábitos alimentares, higiene, sono, álcool, hábitos tabácicos, atividade física, entre outros.

A coleta de dados teve duração de uma semana, sendo esta realizada no mês de dezembro de 2015 e no local de trabalho dos indivíduos.

A partir da coleta, os dados foram tabulados por meio do programa Microsoft Excel (2010) e agrupados por meio da análise descritiva a partir das médias, desvio padrão e percentil. A pesquisa iniciou após aprovação pelo Comitê de Ética em Pesquisa com protocolo número 1.426.275/2015.

\section{Resultados}

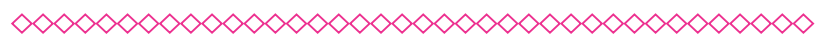

Foram entrevistados 60 funcionários com média de idade de $24 \pm 6,76$ anos, sendo a idade mínima 17 anos e máxima de 50 anos. A maioria dos funcionários eram técnicos e estagiários (95\%) seguidos de docentes (5\%).

Na Tabela 1 são descritos a comparação da percepção da saúde, exercícios físicos e sono entre praticantes e não praticantes de GL.

Observou-se diferença na prática de exercícios físicos, mostrando que os trabalhadores não praticantes de $\mathrm{GL}$ realizam mais frequentemente.

Na Tabela 2 são realizadas as comparações dos hábitos tabácicos e alcoólicos. A principal diferença encontrada foi quanto aos hábitos alcoólicos, mostrando que os indivíduos praticantes bebem mais do que os não praticantes. Além disso, mais da metade dos praticantes não gostariam de reduzir a quantidade de bebida alcoólica consumida.

Quanto aos comportamentos relacionados aos hábitos tabácicos, percebeu-se que 93,3\% de toda a amostra relata não possuir consumo de cigarro.
Na Tabela 3 são abordados os comportamentos alimentares dos pesquisados, onde se verificou que ambos os grupos tomam café da manhã e consumem frutas, legumes e doces, não mostrando resultado significativo.

Apesar disso, constatou-se que mais da metade dos indivíduos praticantes tentam evitar o consumo de alimentos ricos em gordura, caso contrário dos não praticantes.

Por fim, na Tabela 4 são apresentados os dados referentes aos cuidados de saúde, não mostrando diferença entre os grupos.

\section{Discussão}

$\infty \infty \infty \times \infty \times \infty \times \infty \times \infty \times \infty \times \infty \times \infty \times \infty \times \infty \times \infty$

A escolha do tema para realização desse estudo baseou-se na importância da comparação do comportamento em saúde entre praticantes e não praticantes de ginástica laboral, pois são escassos os estudos relacionados ao tema.

A partir da comparação entre os comportamentos de saúde de praticantes e não praticantes de GL esperava-se encontrar grande diferença de comportamentos, tais resultados não foram encontrados.

Uma das hipóteses que pode ser levantada sobre esse evento diz respeito à quantidade de intervenções de $G L$ durante a semana. Para esse estudo, a população praticante de GL tinha duas intervenções por semana. Conforme estudo de Guimarães $^{8}$, quanto maior a intensidade e tempo de intervenção, melhor os resultados obtidos, o que podemos constatar nesta pesquisa, que 0 tempo foi pequeno, acarretando em pequenas diferenças do comportamento dos praticantes em relação aos não praticantes.

Outro aspecto a ser levantado é em relação à abordagem, pois apesar de a GL ser voltada para a promoção da saúde, a educação em saúde acabou não sendo debatida entre os integrantes devido ao curto espaço de tempo, que também pode ser justificado pelo próprio modo de intervenção, pois a Ginástica Laboral visa diminuir riscos de lesão musculoesqueléticas e doenças ocupacionais, como LER/DORT, realizando exercícios preventivos, sem levar o trabalhador ao cansaço, com duração de 10 a 15 minutos 9 .

A educação em saúde se insere na promoção da saúde, sendo esta conceituada como uma 
ferramenta para conduzir pessoas a mudanças, com fins de prevenção e controle de fatores e risco, por meio de hábitos e atitudes saudáveis. Essa mudança no estilo de vida é uma tarefa difícil e de resistência, por isso grande parte da população não consegue realizá-las, mesmo realizando práticas de promoção da saúde, como a GL ${ }^{10}$.

Apesar disso, ainda foram encontrados alguns resultados relevantes e que devem ser discutidos, como a baixa adesão de exercícios físicos tanto de praticantes quanto de não praticantes de $G L, 0$ maior consumo de bebidas alcoólicas de praticantes e o baixo índice de tabagismo entre os pesquisados. Para a baixa adesão da prática de exercícios físicos dos praticantes e não praticantes de $G L$, a hipótese mais coerente segue a linha de que o excesso de trabalho seja um empecilho para sua prática, devido à falta de tempo acarretada por tal. Essa hipótese foi confirmada por Grande e Silva ${ }^{5}$, que afirmam que os trabalhadores têm consciência dos benefícios da prática de atividade física, mas que o trabalho conta como um obstáculo para a mesma, pois após a carga diária de trabalho excessiva os trabalhadores não possuem disposição e tempo para sua realização. Além do mais, Brito e Martins ${ }^{1}$ também afirmam que atualmente as pessoas passam a maior parte do dia no ambiente ocupacional e, muitas vezes, esquecem-se dos cuidados com sua saúde.

Tal fato foi comprovado nessa pesquisa, pois a vontade de iniciar a prática esportiva entre os praticantes de GL foi quase unânime, o que comprova que fatores externos, como a escassez de tempo devido ao trabalho, impede sua realização, ocasionando o sedentarismo na maioria dessa população, expondo-a a doenças decorrentes do mesmo. Esse fato também foi comprovado no estudo de Jerônimo et al. ${ }^{11}$ que observou uma diminuição na prevalência total de atividade física de 5,6\% em 5 anos, o que se torna preocupante no que se refere a saúde da população, considerando as evidências científicas sobre os benefícios da prática regular de exercícios físicos para a saúde física e mental e as consequências da sua ausência.

Os outros achados da presente pesquisa também se relacionam diretamente com a prática regular de exercícios físicos: o tabagismo e consumo de bebidas alcoólicas.

Nessa pesquisa, percebeu-se que índice de tabagismo entre os trabalhadores foi baixo. Tais achados também foram encontrados no estudo de
Carvalho e Moreno ${ }^{12}$ no qual $80 \%$ da população estudada não possuía comprometimento com o tabaco, bem como no estudo de Timossi et al. ${ }^{13}$ onde a prevalência de tabagismo ocorreu em $15 \%$ dos participantes da pesquisa. Em outro estudo, pode-se perceber que estudantes que se declararam fumantes eram sedentários, porém, devido à escassez de estudos nacionais recentes, não foi possível determinar se essas variáveis estão relacionadas ${ }^{13}$.

Com relação ao elevado nível de consumo alcoólico entre os praticantes, pode-se explicar devido à alta carga de estresse que encontram no dia a dia. Além disso, estudos revelam que mesmo baixo, o consumo do álcool pode ter efeitos deletérios, incluindo o desempenho no trabalho, segurança no trânsito e risco de desenvolvimento de doenças crônicas não transmissíveis ${ }^{14}$.

Com os dados dos estudos citados até o presente momento, pode-se relacionar a ausência da prática regular de exercícios físicos com o tabagismo e elevado nível de consumo alcoólico entre praticantes, pois a sua ausência acarreta no sedentarismo e propicia o uso do tabaco e do álcool para diminuir o estresse diário, o que seria solucionado com os exercícios físicos, pois, conforme estudos ${ }^{15,16}$, durante a prática de exercícios físicos ocorre a secreção de hormônios como a endorfina, hormônio responsável pela diminuição da tensão muscular e psicológica, causando sensação de felicidade aos praticantes.

O benefício psicológico ocasionado pela prática de exercícios físicos também foi citado no estudo de Costa, Soares e Teixeira ${ }^{17}$ que afirmam que diversas pesquisas demonstram que a prática regular de exercícios físicos beneficia não apenas fisiologicamente, mas também psicologicamente, ocasionando benefícios como melhora do humor e autoestima, sensação de bem-estar, diminuição da tensão psicológica, redução da ansiedade, dentre outros.

Por fim, vale destacar algumas limitações do estudo. Não foram encontrados na literatura pertinente estudos referentes a esse tema e a essa população, 0 que poderia ter enriquecido a discussão do estudo. Apesar disso, com os resultados obtidos, espera-se contribuir para melhor entendimento da GL no âmbito da educação em saúde, além de auxiliar no planejamento e adequação de programas preventivos ou de atividade física que possam colaborar para a manutenção da saúde dessa população. 
Para isso, é imprescindível que os locais de trabalho reconheçam a necessidade de um trabalho educativo de promoção da saúde para os trabaIhadores, e que estes também assumam suas responsabilidades e compreendam o seu papel na prevenção de agravos, tanto os relacionados ao trabalho como aos hábitos de vida.

\section{Conclusão}

$\infty \infty \infty \times \infty \times \infty \times \infty \times \infty \times \infty \times \infty \times \infty \times \infty \times \infty \times \infty$

Conclui-se que a prática de GL não influenciou os comportamentos em saúde da população envolvida, fazendo-se necessários novos estudos com direcionamento da GL no âmbito da educação em saúde, buscando o cuidado em nível mais abrangente da integralidade.

\section{Referências}

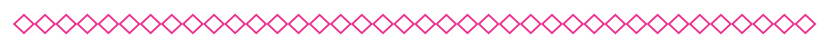

1. Brito ECO, Martins CO. Percepções dos participantes de programa de ginástica laboral sobre flexibilidade e fatores relacionados a um estilo de vida saudável. Rev Bras Prom Saúde 2012;25(4):445-454.

2. Katsurayama M, Parente RCP, Pires ROM. Construção de um modelo teórico dejouriano destinado à avaliação da saúde do trabalhador. Rev Bras Prom Saúde. 2012 Jul/Set; 25(3): 374-380.

3. Manosso M, Lanferdini FJ, Dal'agnol MJ, Roncada C, Dias CP. Comparação dos níveis de estresse e estilo de vida entre praticantes e não praticantes de ginástica laboral. R. Bras. Ci. e Mov. 2014; 22(2): 65-71.

4. World Health Organization. The Ottawa charter for health promotion. Geneve: WHO; 1986.

5. Grande AJ, Silva V. Barreiras e facilitadores para a adesão à prática de atividade física no ambiente de trabalho. 0 mundo da saúde. 2014;38(2):204-209.

6. Rossato LC, Duca GFD, Farias SF, Nahas MV. Prática da ginástica laboral por trabalhadores das indústrias do Rio Grande do Sul, Brasil. Rev. Bras. Educ. Fís. Esporte. 2011; 27(1):15-23
7. Guedes DP, Lopes CC. Validação da versão brasileira do Youth Risk Behavior Survey 2007. Rev Saúde Públ 2010;44(5):840-50.

8. Guimarães LF. Análise de programas de treinamento na musculação prescritos na internet [monografia]. Universidade Federal de Minas Gerais; 2010.

9. Lima V. Ginástica Laboral: atividade física no ambiente de trabalho. $3^{a}$ ed. São Paulo: Phorte Editora; 2008.

10. Souza IVB, Marques DKA, Freitas PES, Lacerda ORM. Educação em saúde e enfermagem: revisão integrativa da literatura. Rev. Ciênc. Saúde Nova Esperança. 2013;11(1):112-121.

11. Jeronimo JS, Jardim VMR, Prado L, Domingues MR. Atividade física em trabalhadores de Centros de Atenção Psicossocial do Sul do Brasil: tendências temporais. Cad. Saúde Públ. 2014; 30(12):2656-2668.

12. Carvalho CMCD, Moreno CRDC. Efeitos de um programa de ginástica laboral na saúde de mineradores. Cad. Saúde Colet. 2007;15 (1):117-30.

13. Timossi LDS, Leite N, Osiecki ANV, Cavazza JF, Cieslak F, Osiecki R. Tabagismo, consumo alcoólico e tempo de sono em trabalhadores ativos da indústria do estado do Paraná-Brasil. Rev. Salud Públ. 2014;16(4):491-504.

14. Santos LRD, Britol ECDC, Netol JCGL, Alves LEP, Alves LRA, Freitas RWJFD. Análise do sedentarismo em estudantes universitários. Rev Enfer UERJ. 2014;22(3):416-21.

15. Domingos JBC, Pillon SC, Santos MAD, Santos RAD, Jora NP, Ferreira PS. Uso de álcool e condições de saúde de motoristas de caminhão. Rev Enfer UERJ. 2014 Nov/Dez;22(6):836-42.

16. Ferreira FG, Bressan J, Marins JCB. Efeitos metabólicos e hormonais do exercício físico e sua atuação sobre a síndrome metabólica. Revista Digital. 2009 Fev;13 (129):1-10.

17. Costa RA, Soares HLR, Teixeira JAC. Benefícios da atividade física e do exercício físico na depressão. Rev. Dep. Psicol. 2007;19(1):273-4. 


\section{Anexos}

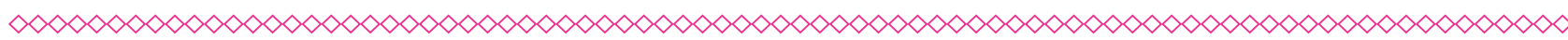

Tabela 1 - Comparação da percepção da saúde, exercícios físicos e sono entre praticantes e não praticantes de GL, 2016

\begin{tabular}{|c|c|c|c|}
\hline Variável & $\begin{array}{l}\text { Praticantes } \\
\text { (n \%) }\end{array}$ & $\begin{array}{l}\text { Não praticantes } \\
\text { (n \%) }\end{array}$ & Total \\
\hline \multicolumn{4}{|l|}{ Saúde } \\
\hline Excelente & 0 & $5(16,6 \%)$ & $5(8,3 \%)$ \\
\hline Muito boa & $10(33,3 \%)$ & $7(23,3 \%)$ & $17(28,3 \%)$ \\
\hline Boa & $15(50 \%)$ & $14(46,6 \%)$ & $29(48,3 \%)$ \\
\hline Razoável & $4(13,3 \%)$ & $4(13,3 \%)$ & $8(13,3 \%)$ \\
\hline Fraca & $1(3,3 \%)$ & 0 & $1(1,66 \%)$ \\
\hline \multicolumn{4}{|l|}{ Relação com a vida } \\
\hline Muito satisfeito & $4(13,3 \%)$ & $4(13,3 \%)$ & $8(13,3 \%)$ \\
\hline $\begin{array}{l}\text { Satisfeito } \\
\text { Insatisfeito } \\
\text { Exercícios físicos nas } 2 \\
\text { últimas semanas }\end{array}$ & $\begin{array}{c}22(73,3 \%) \\
4(13,3 \%)\end{array}$ & $\begin{array}{l}24(80 \%) \\
2(3,3 \%)\end{array}$ & $\begin{array}{c}46(76,6 \%) \\
6(10 \%)\end{array}$ \\
\hline $\begin{array}{l}\text { Todos os dias } \\
7-13 \text { dias } \\
\text { 3-7 dias } \\
\text { Menos de } 3 \text { dias } \\
\text { Não }\end{array}$ & $\begin{array}{c}0 \\
3(10 \%) \\
9(30 \%) \\
10(33,3 \%) \\
8(26,6 \%)\end{array}$ & $\begin{array}{c}6(20 \%) \\
3(10 \%) \\
2(6,6 \%) \\
9(30 \%) \\
10(33,3 \%)\end{array}$ & $\begin{array}{c}6(10 \%) \\
6(10 \%) \\
11(18,3 \%) \\
19(31,6 \%) \\
18(30 \%)\end{array}$ \\
\hline $\begin{array}{l}\text { Desejo de aumentar a } \\
\text { prática } \\
\text { Sim } \\
\text { Não }\end{array}$ & $\begin{array}{c}26(86,6 \%) \\
4(13,3 \%)\end{array}$ & $\begin{array}{l}27(90 \%) \\
3(10 \%)\end{array}$ & $\begin{array}{c}53(88,3 \%) \\
7(11,6 \%)\end{array}$ \\
\hline $\begin{array}{l}\text { Horas diárias de sono } \\
4 \text { horas } \\
4-6 \text { horas } \\
6-8 \text { horas } \\
8 \text { horas }\end{array}$ & $\begin{array}{c}0 \\
13(43,3 \%) \\
15(50 \%) \\
2(6,6 \%)\end{array}$ & $\begin{array}{l}2(6,6 \%) \\
8(26,6 \%) \\
15(50 \%) \\
5(16,6 \%)\end{array}$ & $\begin{array}{l}2(3,3 \%) \\
21(35 \%) \\
30(50 \%) \\
7(11,6 \%)\end{array}$ \\
\hline $\begin{array}{l}\text { Cansaço ao acordar } \\
\text { Sempre } \\
\text { Nunca } \\
\text { Muitas vezes } \\
\text { Poucas vezes }\end{array}$ & $\begin{array}{c}6(20 \%) \\
1(3,3 \%) \\
9(30 \%) \\
14(46,6 \%)\end{array}$ & $\begin{array}{c}5(16,6 \%) \\
4(13,3 \%) \\
7(23,3 \%) \\
14(46,6 \%)\end{array}$ & $\begin{array}{c}11(18,3 \%) \\
5(8,3 \%) \\
16(26,6 \%) \\
28(46,6 \%)\end{array}$ \\
\hline
\end{tabular}

Fonte: Elaborado pelos autores 
Tabela 2 - Comparação dos hábitos tabácicos e alcoólicos de praticantes e não praticantes de GL, 2016

\begin{tabular}{|c|c|c|c|}
\hline Variável & $\begin{array}{l}\text { Praticantes } \\
\text { (n \%) }\end{array}$ & $\begin{array}{l}\text { Não praticantes } \\
(\mathrm{n} \%)\end{array}$ & Total \\
\hline \multicolumn{4}{|l|}{ Hábitos tabácicos } \\
\hline $\begin{array}{l}\text { Não fumo } \\
\text { Fumo, média de menos } \\
\text { de um cigarro por dia }\end{array}$ & $\begin{array}{c}28(93,3 \%) \\
2(6,6 \%)\end{array}$ & $\begin{array}{c}28(93,3 \%) \\
1(3,3 \%)\end{array}$ & $\begin{array}{c}56(93,3 \%) \\
3(5 \%)\end{array}$ \\
\hline $\begin{array}{l}\text { Fumo de } 1 \text { a } 10 \text { cigarros } \\
\text { por dia }\end{array}$ & 0 & $1(3,3 \%)$ & $1(1,6 \%)$ \\
\hline \multicolumn{4}{|l|}{ Pretende reduzir } \\
\hline $\begin{array}{l}\text { Sim } \\
\text { Não }\end{array}$ & $\begin{array}{c}1(3,3 \% \%) \\
1(3,3 \%)\end{array}$ & $\begin{array}{c}2(6,6 \%) \\
0\end{array}$ & $\begin{array}{c}3(5 \%) \\
1(1,6 \%)\end{array}$ \\
\hline \multicolumn{4}{|l|}{ Hábitos alcoólicos } \\
\hline Nunca bebi & $3(10 \%)$ & $5(16,6 \%)$ & $8(13,3 \%)$ \\
\hline $\begin{array}{l}\text { Bebo apenas em situações } \\
\text { especiais }\end{array}$ & $7(23,3 \%)$ & $16(53,3 \%)$ & $23(38,3 \%)$ \\
\hline Bebo ocasionalmente & $15(50 \%)$ & $7(23,3 \%)$ & $22(36,6 \%)$ \\
\hline Bebo regularmente & $5(16,6 \%)$ & $12(40 \%)$ & $17(28,3 \%)$ \\
\hline
\end{tabular}

\section{Consumo alcoólico nas 2} últimas semanas

7-13 dias

3-7 dias

Menos de 3 dias

Não

$\begin{array}{ccc}0 & 2(6,6 \%) & 2(3,3 \%) \\ 8(26,6 \%) & 4(13,3 \%) & 12(20 \%) \\ 13(43,3 \%) & 5(16,6 \%) & 18(30 \%) \\ 1(3,3 \%) & 6(20 \%) & 7(11,6 \%)\end{array}$

\section{Pretende reduzir}

\begin{tabular}{lccc} 
Sim & $2(6,6 \%)$ & 0 & $2(3,3 \%)$ \\
Não & $19(63,3 \%)$ & $13(43,3 \%)$ & $32(53,3 \%)$ \\
\hline
\end{tabular}

Fonte: Elaborado pelos autores 
Tabela 3 - Comparação dos hábitos alimentares entre praticantes e não praticantes de GL, 2016

\section{Variável}

\section{Café da manhã}

Diariamente

Quase todos os dias

Às vezes

Raramente ou nunca

Evitar consumo alimentos

\section{ricos em gordura}

Sim

Não

\section{Consumo de frutas}

Pelo menos uma vez por dia

Uma vez em cada 2/3 dias

Cerca de uma vez por semana

Menos de uma vez por semana

Nunca

\section{Consumo de legumes}

Pelo menos uma vez por dia

Uma vez em cada $2 / 3$ dias

Cerca de uma vez por semana

Menos de uma vez por semana

Nunca

\section{Consumo de doces}

Pelo menos uma vez por dia

Uma vez em cada $2 / 3$ dias

Cerca de uma vez por semana

Menos de uma vez por semana
Praticantes

(n \%)

$17(56,6 \%)$
$3(10 \%)$
$4(13,3 \%)$
$6(20 \%)$

$16(53,3 \%)$

$14(46,6 \%)$

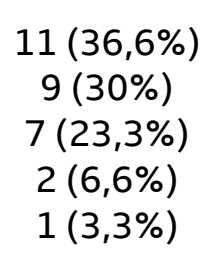
$13(43,3 \%)$
$10(33,3 \%)$
$4(13,3 \%)$
$2(6,6 \%)$
$1(3,3 \%)$

$10(33,3 \%)$
$10(33,3 \%)$
$5(16,6 \%)$
$5(16,6 \%)$

$11(36,6 \%)$

$19(63,3 \%)$

$$
\begin{gathered}
16(53,3 \%) \\
4(13,3 \%) \\
5(16,6 \%) \\
5(16,6 \%)
\end{gathered}
$$

\section{Total}

$33(55 \%)$

$7(11,6 \%)$

$9(15 \%)$

$11(18,3 \%)$

$27(45 \%)$

$33(55 \%)$

$25(41,6 \%)$

$16(26,6 \%)$

$15(25 \%)$

$6(10 \%)$

$1(1,6 \%)$

$26(43,3 \%)$

$18(30 \%)$

$10(16,6 \%)$

$4(6,6 \%)$

$2(3,3 \%)$
$15(25 \%)$

$20(33,3 \%)$

$17(28,3 \%)$

$8(13,3 \%)$

Fonte: Elaborado pelos autores

(clique para voltar ao texto) 
Tabela 4 - Comparação dos cuidados de saúde entre praticantes e não praticantes de GL, 2016

\section{Variável}

Escova os dentes

Três ou mais vezes ao dia

Duas vezes por dia

\section{Vai anualmente ao}

dentista

Sim

Não

\section{Faz checkup anualmente}

Sim

Não

\section{Não praticantes} (n \%)

$24(76,6 \%)$

$6(23,3 \%)$

$26(86,6 \%)$

$4(13,3 \%)$

$50(83,3 \%)$

$10(16,6 \%)$

\section{Total}

$23(73,3 \%)$

$7(26,6 \%)$

$$
26(86,6 \%)
$$

$49(81,6 \%)$

$4(13,3 \%)$

$11(18,3 \%)$

$14(46,6 \%)$

$16(53,3 \%)$
$26(43,3 \%)$

$34(56,6 \%)$

\section{Filtro solar}

Sempre

$24(76,6 \%)$

$24(80 \%)$

$48(80 \%)$

Regularmente

Normalmente não

Nunca

0

$3(10 \%)$

$9(15 \%)$

$1(3,3 \%)$

$1(1,6 \%)$

0

$2(6,6 \%)$

$2(3,3 \%)$

Fonte: Elaborado pelos autores

(clique para voltar ao texto) 